EPJ Web of Conferences 113, 08004 (2016)

DOI: $10.1051 /$ epjconf/201611308004

CC Owned by the authors, published by EDP Sciences, 2016

\title{
Helium atom under pressure
}

\author{
Jérémy Dohet-Eraly ${ }^{1, a}$ and Daniel Baye ${ }^{2, b}$ \\ ${ }^{1}$ TRIUMF, 4004 Wesbrook Mall, Vancouver BC V6T 2A3, Canada \\ ${ }^{2}$ Physique Quantique, and Physique Nucléaire Théorique et Physique Mathématique, C.P. 229, Université \\ libre de Bruxelles (ULB), 1050 Brussels, Belgium
}

\begin{abstract}
Hard-sphere confinement is used to study helium atoms under pressure. The confined-helium Schrödinger equation is solved with a high accuracy by a Lagrangemesh method.
\end{abstract}

The effects of high pressure on a helium gas can be estimated by studying the helium atom in a hard confinement, i.e. confined at the centre of an impenetrable spherical cavity, for different cavity radii. Contrary to the free helium atom, the confined helium has not been described with a very high accuracy until recently, when we have developed a Lagrange-mesh method to study this system [1]. This method improves by several order of magnitudes the accuracy of previous approaches [2-4].

The outlines of the model are the following. The assumed-infinite-mass nucleus is fixed and the electrons are characterized by coordinates $\boldsymbol{r}_{1}$ and $\boldsymbol{r}_{2}$ with respect to this nucleus. In atomic units, the Hamiltonian of the helium atom reads

$$
H=-\frac{1}{2} \Delta_{1}-\frac{1}{2} \Delta_{2}-\frac{2}{r_{1}}-\frac{2}{r_{2}}+\frac{1}{r_{12}},
$$

where $\boldsymbol{r}_{12}=\boldsymbol{r}_{1}-\boldsymbol{r}_{2}$ and $\Delta_{1}$ and $\Delta_{2}$ are the Laplacians with respect to $\boldsymbol{r}_{1}$ and $\boldsymbol{r}_{2}$. The confinement is introduced by forcing the wave function into some spherical cavity of radius $R\left(r_{1}, r_{2} \leq R\right)$. The wave function $\psi\left(r_{1}, r_{2}, r_{12}\right)$ of an $S$ state must thus verify the Schrödinger equation

$$
H \psi\left(r_{1}, r_{2}, r_{12}\right)=E \psi\left(r_{1}, r_{2}, r_{12}\right)
$$

and vanishes at $r_{1}=R$ and $r_{2}=R$. The coordinates $\left(r_{1}, r_{2}, r_{12}\right)$ are advantageously replaced by the coordinates $(u, v, w)$ defined over $[0,1]$ by

$$
u=\frac{r_{1}-r_{2}+r_{12}}{2 R-r_{1}-r_{2}+r_{12}}, v=\frac{-r_{1}+r_{2}+r_{12}}{2 R-r_{1}-r_{2}+r_{12}}, w=\frac{r_{1}+r_{2}-r_{12}}{2 R} .
$$

The confinement implies that the wave function $\psi(u, v, w)$ vanishes at $u=1, v=1$, and $w=1$.

The Schrödinger equation is solved by the Lagrange-mesh method [5-7], an approximate variational approach taking the form of a system of mesh equations by computing the Hamiltonian and overlap matrix elements with a Gauss quadrature. Using the coordinates $(u, v, w)$ is essential for an easy treatment of the confinement and an high accuracy of the Gauss quadrature. The ground-state energy and wave function are obtained by diagonalizing a rather large (matrix dimension $\approx 10^{3} \sim 2 \times 10^{4}$ ) but sparse matrix. Ground-state energies and mean interparticle distances for several confinement radii $R$ are given in table 1. The pressure acting on the confined helium atom is also given in table 1. It is

\footnotetext{
a e-mail: jdoheter@triumf.ca

be-mail: dbaye@ulb.ac.be
} 
Table 1. Ground-state energy and mean interparticle distances of a helium atom confined in a sphere of radius $R$ and pressure acting on such a confined helium atom [1]. Atomic units are used. The powers of ten are indicated between brackets.

\begin{tabular}{rllll}
\hline$R$ & \multicolumn{1}{l}{ E } & $\left\langle r_{12}\right\rangle$ & $\left\langle r_{1}\right\rangle=\left\langle r_{2}\right\rangle$ & $P$ \\
\hline 0.1 & 906.562422919888 & 0.0695803828842 & 0.0495012463401 & $1.50742673864[5]$ \\
1.0 & 1.0157549760484 & 0.643664253878 & 0.4417966321033 & $9.5100856621[-1]$ \\
10.0 & -2.903724375687 & 1.422070172936 & 0.929472251212 & $2.711[-12]$ \\
\hline
\end{tabular}

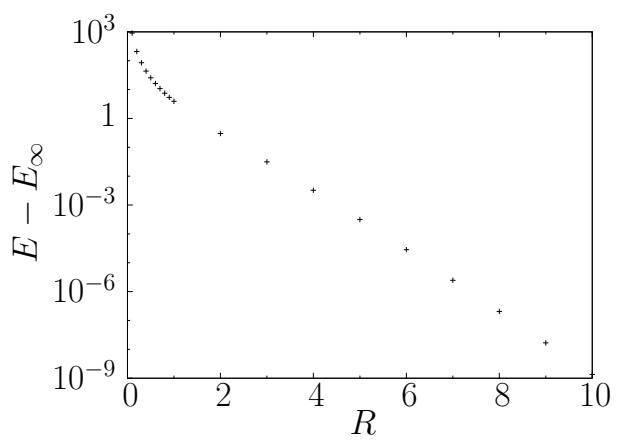

Figure 1. Difference between the ground-state energies of confined helium $(E)$ and of free helium $\left(E_{\infty}\right)$ as a function of the confinement radius $R$. Atomic units are used.

calculated from the radius dependence of the energies $E(R)$ by the formula [2-4],

$$
P=-\frac{1}{4 \pi R^{2}} \frac{d E}{d R}
$$

where the derivative is evaluated by a finite-difference formula. A similar accuracy is obtained for the energy and interparticle distances of the first excited singlet level and the lowest triplet level [1]. Although the Lagrange-mesh method can be applied easily for small and large radii, it is particularly efficient for small radii (smaller variational basis size and better accuracy). The dependence of the ground-state energy on the confinement radius $R$ is shown graphically in Fig. 1. For large confinement radii $(R \gtrsim 2 \mathrm{fm})$, the confined helium energy tends nearly exponentially to the free helium energy. A parametrization of this curve could allow an easy evaluation of the pressure at very large radii, difficult to reach by direct calculations.

\section{Acknowledgements}

This text presents research results of the interuniversity attraction pole programme P7/12 initiated by the Belgianstate Federal Services for Scientific, Technical and Cultural Affairs. TRIUMF receives funding via a contribution through the National Research Council Canada.

\section{References}

[1] D. Baye, J. Dohet-Eraly, Phys. Chem. Chem. Phys. (2015), DOI:10.1039/C5CP00110B

[2] N. Aquino, A. Flores-Riveros, J. Rivas-Silva, Phys. Lett. A 307, 326 (2003)

[3] C. Laughlin, S.I. Chu, J. Phys. A 42, 265004 (2009)

[4] S. Bhattacharyya, J.K. Saha, P.K. Mukherjee, T.K. Mukherjee, Phys. Scripta 87, 065305 (2013)

[5] D. Baye, P.-H. Heenen, J. Phys. A 19, 2041 (1986)

[6] M. Hesse, D. Baye, J. Phys. B 32, 5605 (1999)

[7] D. Baye, Phys. Rep. 565, 1 (2015) 